\title{
Scaling behaviour of relaxation dependences in metaloxide superconductors
}

\author{
A S SIDORENKO, G I PANAITOV, A M GABOVICH*, D P \\ MOISEEV* and V M POSTNIKOV* \\ Institute of Applied Physics, Mold. Academy of Sciences, 277028, Kishinev, USSR \\ *Institute of Physics, Ukr. Academy of Sciences, 252650, Kiev, USSR
}

In a variety of experiments (see, e.g. Muller et al 1987, Havronin et al 1988) it was shown that the superconducting glass state is realized in granular superconductors (Josephson media) for fields $H \ll H_{c l}\left(H_{c l}\right.$ is a first single-crystal critical field). These state properties are determined by the frustration of weak superconducting links. In particular, a slow logarithmic relaxation of the thermoremanent magnetization is observed

$$
M(t)=M_{0}-S_{\text {int }}
$$

In some cases, the dependence of the relaxation rate $S=-\mathrm{d} M / d_{\mathrm{int}}$ on temperature and external magnetic field were investigated. For instance, it was discovered that for powder samples of $\mathrm{La}-\mathrm{Sr}-\mathrm{Cu}-\mathrm{O}$ system the power-law dependence occurs: $S \sim H^{3}$ (Mota et al 1987).

In the present work we have measured the magnetization relaxation at $T=4 \cdot 2 \mathrm{~K}$ in two substantially different superconductors: $\mathrm{BaPb}_{0.75} \mathrm{Bi}_{0.25} \mathrm{O}_{3}\left(\mathrm{BPB}, T_{\mathrm{c}} \approx 10 \mathrm{~K}\right.$, $\left.H_{c 1} \approx 12 \mathrm{Oe}\right)$ and $\mathrm{YBa}_{2} \mathrm{Cu}_{3} \mathrm{O}_{7-x}\left(\mathrm{YBCO}, T_{c} \approx 92 \mathrm{~K}\right)$. The SQUID magnetometer with active and passive electromagnetic field shielding guaranteed measurements for $H$ up to $900 \mathrm{Oe}$. The polycrystalline specimens were prepared by the solid-state synthesis method.

It is difficult to determine the precise value of $H_{c 1}$ for the YBCO ceramics. But, according to literature, $H_{c 1}$ is between $300 \mathrm{Oe}$ and $1500 \mathrm{Oe}$, the discrepancy being due to the strong anisotropy of the superconducting properties of metaloxides YBCO (Shelton 1987).

Dependences of $S$ and the trapped magnetic flux $M_{0}$ under the field-cooled (FC) condition on the magnitude of the external magnetic field $H(5+600 \mathrm{Oe})$ were determined. In BPB samples for fields $H \gtrsim H_{c 1}$ the curves $S(H)$ and $M_{0}(H)$ started to saturate, whereas in YBCO ceramics the saturation of $S(H)$ and $M_{0}(H)$ was not achieved for accessible fields.

At first sight the experimental data differ greatly. But the results can be represented as the dependences of $S$ on $M_{0}$. Then, as can be readily seen from figure 1 , the experimental data for various samples follow the universal scaling dependence $\ln S=k \ln M_{0}$ with the exponent $K \approx 3$. This agrees with the results of Mota et al (1987), if one suggests that $M_{0}$ is proportional to $H$ in Mota et al (1987), where the isothermal conditions have been used.

The observed universal dependence $S \sim M_{0}^{3}$ is probably a manifestation of the 


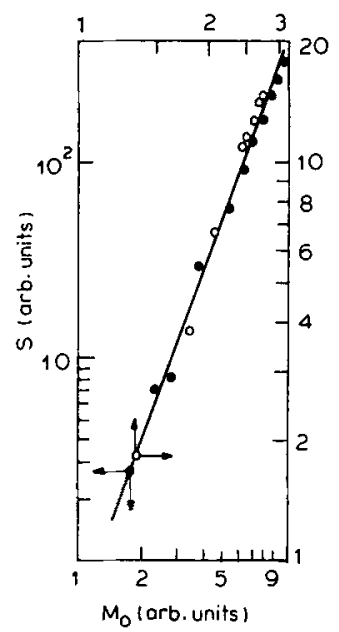

Figure 1. Scaling law for relaxation rate $S=\mathrm{d} M / \mathrm{d}_{\mathrm{int}}(\bullet \mathrm{YBCO}, \bigcirc \mathrm{BPB})$.

superconducting glass state in porous ceramics. It is interesting to note that the observed behaviour does not depend on the type of weak links between superconducting grains (S-I-S links in BPB or S-N-S links in YBCO) and critical current magnitudes which are quite different for the samples studied.

Scaling law for relaxation rate $S=-\mathrm{d} M / \mathrm{d}_{\text {int }}$

\section{References}

Havronin V P, Luzianin I D and Ginzburg S L 1988 Preprint N1370 Leningrad Institute of Nuclear Physics, Leningrad

Müller K A, Takashige M and Bednorz J G 1987 Phys. Rev. Lett. 581143

Mota A S et al 1987 Phys. Rev. B36 4011

Shelton R N 1987 J. Mod. Phys. B1 401 